\title{
How well can we predict the total cross section at the LHC?
}

\author{
P V Landshoff \\ Department of Applied Mathematics and Theoretical Physics \\ University of Cambridge \\ Cambridge CB3 OWA \\ England
}

\begin{abstract}
Independently of any theory, the possibility that the large value of the Tevatron cross section claimed by CDF is correct suggests that the total cross section at the LHC may be large. Because of the experimental and theoretical uncertainities, the best prediction is $125 \pm 35 \mathrm{mb}$.
\end{abstract}

PACS: $13.85 . \mathrm{Lg}$ 13.85.Dz 13.60.Hb 11.55.Jy

This talk is based on work with Polkinghorne, Donnachie, Nachtmann and others going back to 1970. Further details may be found in our book[1]. While theoretical understanding of long-range strong interactions has increased greatly since then, it is still not good enough to allow a confident prediction of even the value of the total cross section at the LHC. When I prepared this talk, I quoted $125 \pm 25 \mathrm{mb}$, but at the meeting Alan Martin predicted $90 \mathrm{mb}$.

Alan Martin's prediction is viable only if one believes that the CDF measurement[2] of the $\bar{p} p$ cross section at the Tevatron is wrong. This is the upper of the $\sqrt{s}=1800$ Gev data points shown in figure 1 . The curves in the figure are based on $\rho, \omega, f_{2}, a_{2}$ and soft-pomeron exchange, and they go nicely through the E710 Tevatron data point[3]. At $\sqrt{s}=14 \mathrm{TeV}$ only the soft-pomeron term $21.7 s^{0.0808}$ survives, giving a prediction of $101.5 \mathrm{mb}$.

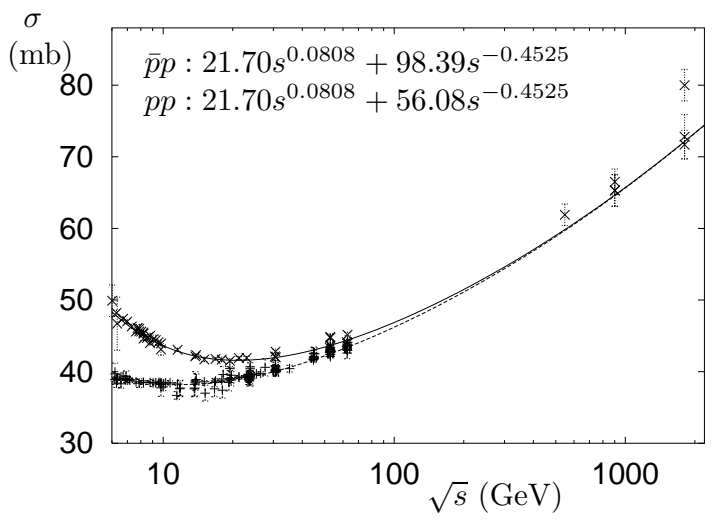

Figure 1: $p p$ and $\bar{p} p$ total cross sections

A significant discovery at HERA was that soft-pomeron exchange does not describe the rise at small $x$ of the proton structure function $F_{2}\left(x, Q^{2}\right)$. That is, a term that behaves 
as $(1 / x)^{\varepsilon_{1}}$ with $\varepsilon_{1} \approx 0.08$ is not sufficient. As $Q^{2}$ increases, the data behave more and more as $(1 / x)^{\varepsilon_{0}}$ with $\varepsilon_{0} \approx 0.4$. The simplest description of the data at very small $x$ is

$$
F_{2}\left(x, Q^{2}\right)=f_{0}\left(Q^{2}\right) x^{-\varepsilon_{0}}+f_{1}\left(Q^{2}\right) x^{-\varepsilon_{1}}
$$

As is well known, but is usually ignored, there are significant mathematical difficulties in the usual perturbation-theory application of DGLAP evolution at small $x$. Applying DGLAP evolution to a power fit such as (1) gives differential equations for the coefficient functions $f_{0}\left(Q^{2}\right)$ and $f_{1}\left(Q^{2}\right)$. However, only the one for $f_{0}\left(Q^{2}\right)$ is valid: because $\varepsilon_{1}$ is small, perturbation theory breaks down for $f_{1}\left(Q^{2}\right)$. If one extracts $f_{0}\left(Q^{2}\right)$ from fitting the small- $x$ data, it agrees with the solution to the differential equation astonishingly well, in NLO and even in leading order[1].

So it is natural to include also a hard-pomeron-exchange term $s^{\varepsilon_{0}}$ in the fits to the $p p$ and $\bar{p} p$ total cross sections[4]. Depending on how large one makes the contribution from this term, one can make the fit go through the CDF data point, or anywhere between the $\mathrm{CDF}$ and $\mathrm{E} 710$ points. Making it go through the CDF point leads to a prediction of about $160 \mathrm{mb}$ for the LHC total cross section.

This highlights the issue that is generally referred to as "unitarity", which can mean various things. One is the Froissart-Martin-Lukaszuk bound, that at large enough $s$

$$
\sigma^{T O T}<\frac{\pi}{m_{\pi}^{2}} \log ^{2}\left(s / s_{0}\right)
$$

For reasonable values of the unknown scale $s_{0}$ this gives a bound of several barns, so it is not really relevant. A more stringent condition is obtained by writing the elasticscattering amplitude in so-called eikonal form:

$$
A\left(s,-\mathbf{q}^{2}\right)=2 i s \int d^{2} b e^{-i \mathbf{q} \cdot \mathbf{b}}\left(1-e^{-\chi(s, b)}\right)
$$

Then a constraint from unitarity is that $\operatorname{Re} \chi(s, b) \geq 0$.

A much more difficult consequence of unitarity is that if it is possible to exchange an object such as the soft pomeron, one must also take account of the exchange of two or more of them. While we know certain general features of these further exchanges, we do not know how to make quantitative calculations. One model is to expand the exponential in (3) as a power series:

$$
A\left(s,-\mathbf{q}^{2}\right)=2 i s \int d^{2} b e^{-i \mathbf{q} \cdot \mathbf{b}}\left(\chi-\frac{\chi^{2}}{2 !}+\frac{\chi^{3}}{3 !} \ldots-\frac{(-\chi)^{n}}{n !} \ldots\right)
$$

and identify the first term as the contribution from the single pomeron exchange. The second term then has the correct features of the exchange of two pomerons, the third term of three, and so on. But this is only a model: there is no reason to believe that it is correct, and various reasons to believe that it is not[1]. 

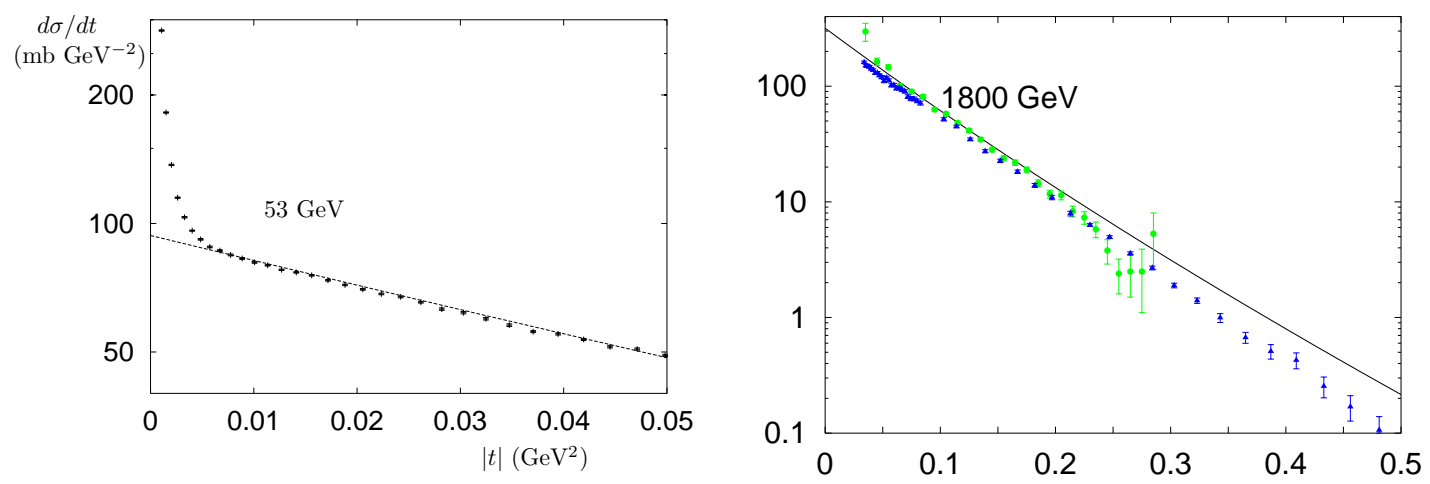

Figure 2: elastic scattering $-p p$ at $53 \mathrm{GeV}$ and $\bar{p} p$ at $1800 \mathrm{GeV}$

The left hand part of figure 2 shows that, beyond the Coulomb peak, single pomeron exchange gives an excellent fit to the $p p$ elastic-scattering differential cross section at small and medium values of $t$. The right-hand part of the figure shows that, at a rather higher energy, the fit is good only at relatively small values of $t$. It is known that adding in the contribution from the exchange of two pomerons should bend the curve downwards. I now describe a very crude way to calculate this.

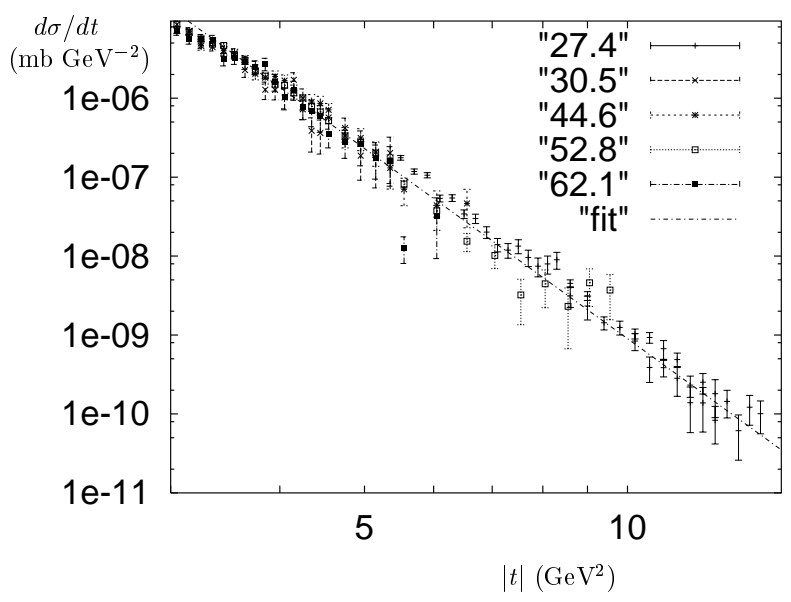

Figure 3: $p p$ elastic scattering at large $t$. The line is $0.09 t^{-8}$.

First, at large $t$, the data for $p p$ elastic scattering are independent of energy and fit well to $d \sigma / d t \sim t^{-8}$. See figure 3. This behaviour is just what one gets from calculating the exchange of 3 gluons to lowest order in perturbative QCD. There is evidence that this same mechanism contributes to the creation of the dips seen in figure 4 . To understand this, note first that there are rather general principles that relate the phase of an elastic amplitude to its energy dependence at that value of $t$. From this one knows that, near the dip, the amplitude is neither close to being real nor imaginary. This means that it is something of a coincidence that indeed there is a dip: there has to be destructive interference in both the real and the imaginary parts of the amplitude at the same value of $t$. The simplest way to achieve this is to cancel the imaginary parts of single-pomeron and two-pomeron exchange, and use 3-gluon exchange (which is real) to cancel the real 
parts. Pomeron exchange is $C=+1$ exchange and so does not change if we replace one of the initial protons with an antiproton, but 3-gluon exchange changes sign because it is $C=-1$. So if 3-gluon exchange helps to give a dip in $p p$ scattering, it cannot do so in $\bar{p} p$ scattering. And indeed experiment finds that $\bar{p} p$ scattering does not have a dip at $\sqrt{s}=53 \mathrm{GeV}$.
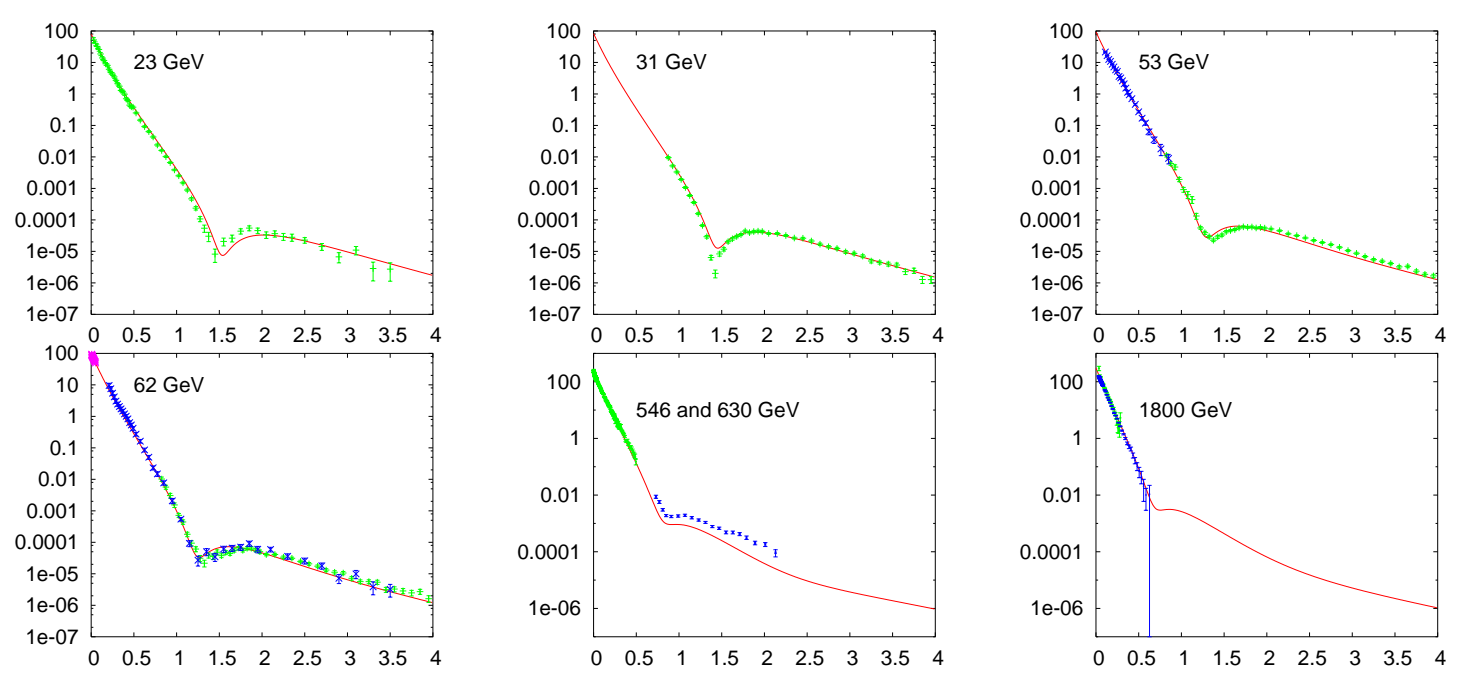

Figure 4: $p p$ and $\bar{p} p$ elastic scattering data, with a crude model calculation.

So I have constructed a crude model, whose output is the curves in figure 4 and which is an adaptation of (4):

$$
A\left(s,-\mathbf{q}^{2}\right)=2 i s \int d^{2} b e^{-i \mathbf{q} \cdot \mathbf{b}}\left(\chi-\frac{\lambda \chi^{2}}{2 !}\right)
$$

I took $\chi(s, b)$ to correspond to the sum of the single exchanges of $\rho, \omega, f_{2}, a_{2}$ and the soft and hard pomerons. The parameter $\lambda$ determines the strength of the double exchange and is chosen so as to cancel the imaginary part of the amplitude at the dip. The 3-gluon exchange term also includes a parameter that switches off its large- $t$ behaviour, $t^{-4}$, so that it does not diverge at $t=0$.

The result is that the power behaviour of the total cross ection from single exchange is damped by the double exchange, and the extrapolation to LHC energy is pulled down from $160 \mathrm{mb}$ to $125 \mathrm{mb}$. Clearly, this model is very crude, but it is the best that can be done at present.

\section{REFERENCES}

1. A Donnachie, H G Dosch, P V Landshoff and O Nachtmann, Pomeron Physics and QCD, Cambridge University Press (2002)

2. F Abe et al, CDF Collaboration, Physical Review D50 (1994) 5550

3. N A Amos et al, E710 Collaboration, Physical Review Letters 63 (1989) 2784

4. A Donnachie and P V Landshoff, Physics Letters B595 (2004) 393 